\title{
Fracionamento e germinação de sementes de uvaia (Eugenia pyriformis Cambess. - Myrtaceae)
}

\author{
CRISTIANA V. SILVA ${ }^{1}$, DENISE A.C. BILIA ${ }^{1}$, ANGELA M. MALUF ${ }^{1}$ e CLAUDIO J. BARBEDO ${ }^{1,2}$
}

(recebido: 25 de julho de 2002; aceito: 6 de março de 2003)

\begin{abstract}
Seed germination of "uvaia" (Eugenia pyriformis Cambess. - Myrtaceae) after cutting). Eugenia pyriformis Cambess. ("uvaia"), a tropical tree species from Brazil, could be used in forest regeneration programs, arboriculture and for fruit industrial use. However, seed production is not enough to produce seedlings in commercial scale. In this work, when seeds of E. pyriformis were longitudinally or transversally sectioned, high percentage of germination was maintained and even those that had been cut to leave $1 / 4^{\text {th }}$ of the original seed size have germinated and produced normal seedlings. In a second experiment, seeds were cut into eight parts and at least one of them germinated. Our results showed that it is possible to obtain more than one normal seedling from each seed. Furthermore, higher germination percentage and normal seedling growth have been obtained when seeds were cut in the longitudinal way.
\end{abstract}

Key words - Myrtaceae, propagation, seed germination

RESUMO - (Fracionamento e germinação de sementes de uvaia (Eugenia pyriformis Cambess. - Myrtaceae)). Eugenia pyriformis Cambess. (uvaia), espécie arbórea tropical do Brasil, pode ser utilizada em programas de reflorestamento e em áreas urbanas e seus frutos apresentam potencialidade de uso industrial. Contudo, apesar da elevada porcentagem de germinação, a produção de sementes dessa espécie é insuficiente para produção de mudas em escala comercial. No presente trabalho, sementes de E. pyriformis foram cortadas longitudinalmente ou transversalmente em duas partes e mantiveram a elevada porcentagem de germinação e de produção de plântulas normais, mesmo quando a fração resultante do corte tinha apenas $1 / 4$ da semente. Em um segundo experimento, as sementes foram cortadas em até oito partes e pelo menos uma das frações de cada semente continuou apresentando germinação. Os resultados mostraram que, através do fracionamento, é possível obter mais de uma plântula normal a partir de cada semente. Os melhores resultados foram obtidos com o corte longitudinal.

Palavras-chave - Germinação de sementes, Myrtaceae, propagação

\section{Introdução}

A família Myrtaceae possui várias espécies que produzem frutos comestíveis de sabor agradável, como goiaba, jabuticaba, araçá, guabiroba, cagaita e cambuci, além de terem características adequadas ao uso na arborização urbana (Correa 1975, Silva et al. 2001). Algumas, como Eugenia uniflora L., apresentam vantagens sobre outras frutíferas cultivadas por serem mais eficientes no acúmulo de nutrientes nos frutos, principalmente nos cultivos em solos de baixa fertilidade (Nascimento et al. 1995).

Algumas espécies de Myrtaceae são utilizadas como plantas medicinais no Paraguai e Argentina, formando um complexo conhecido popularmente como Ñangapary (Schmeda-Hirschmann et al. 1987 , Consolini et al. 1999). Dentre elas, as cerca de 400 do gênero Eugenia assumem destaque especial.

\footnotetext{
1. Instituto de Botânica, Caixa Postal 4005, 01061-970 São Paulo, SP, Brasil.

2._Autor para correspondência: cbarbedo@ibot.sp.gov.br
}

Embora em algumas espécies popularmente consideradas medicinais não se tenha cientificamente verificada tal propriedade (Pepato et al. 2001), em outras há pesquisas confirmando a presença de substâncias reconhecidamente potenciais para o uso medicinal. A decocção de folhas de Eugenia uniflora em água, por exemplo, pode servir para controle da hipertensão, diminuição do colesterol e ácido úrico, emagrecimento e, também, como adstringente e digestivo (Schmeda-Hirschmann et al. 1987). Em folhas e caules de E. moraviana Berg. foi isolado o ácido $6 \alpha$-hidroxibetulínico (um triterpeno), o ácido platânico, o ácido betulínico e o $\beta$-sitosterol, compostos que tem atraído muita atenção pelo seu potencial de uso no tratamento de HIV, tumores, malária e processos inflamatórios (Lunardi et al. 2001). Nas folhas de E. uniflora e, principalmente, nas de E. pyriformis Cambess., há flavonóides com propriedades inibidoras da xantino-oxidase, atuando no tratamento da gota humana (Schmeda-Hirschmann et al. 1987, Theoduloz et al. 1988).

Eugenia pyriformis Cambess. é espécie arbórea nativa da região Sul do Brasil (Mattos 1954) e pode ser 
encontrada desde São Paulo até o Rio Grande do Sul. A semente não apresenta endosperma, ou seja, é exalbuminosa (Flores \& Rivera 1989). O seu embrião é eugenióide (cotilédones carnosos e radícula inconspícua) e, como nas demais espécies brasileiras de Eugenia, é aparentemente indiviso. Devido à fusão parcial ou total dos cotilédones, é chamado pseudomonocotilenar (Gurgel \& Soubihe Sobrinho 1951, McVaugh 1956, Landrum \& Kawasaki 1997, Anjos \& Ferraz 1999, Kawasaki 2000). Essa característica auxiliou, inclusive, a separação do gênero Syzygyium de Eugenia, inicialmente considerados todos neste último (Schmid 1972).

Ainda há dificuldades na produção de mudas de Eugenia pyriformis pela carência de sementes. $\mathrm{Na}$ literatura só foram encontrados registros de pomares experimentais, mas nenhum implantado para produção de sementes ou para produção comercial de frutos. Além disso, há poucas sementes por fruto (Mattos 1954) e a dificuldade na produção de mudas é ainda maior pela falta de tecnologia que permita maximizar o uso das sementes, principalmente quanto à sua conservação e multiplicação. Sementes de várias espécies de Eugenia apresentam baixa longevidade natural (Rizzini 1970, Von Bülow et al. 1994, Gentil \& Ferreira 1999). O emprego de técnicas adequadas de armazenamento, como as obtidas por Von Bülow et al. (1994) para sementes de E. calycina Cambess. e por Barbedo et al. (1998) para sementes de E. involucrata DC., pode ampliar o potencial de armazenamento das sementes de espécies do gênero Eugenia. A longevidade natural das sementes de E. pyriformis, contudo, ainda é curta. Essas características tornam necessários estudos para maximizar o uso das sementes que se obtém a cada safra.

A família Myrtaceae contempla algumas espécies com sementes poliembriônicas (Landrum \& Kawasaki 1997), inclusive dentro do gênero Eugenia (Johnson 1936, Gurgel \& Soubihe Sobrinho 1951, Lughadha \& Proença 1996). Contudo, não há evidências da presença de poliembrionia em uvaia (Gurgel \& Soubihe Sobrinho 1951). A presença de poliembrionia em diversas espécies da família Myrtaceae e, ainda, de embrião aparentemente indiviso em Eugenia sugerem a possibilidade de se aumentar o número de plântulas obtidas de cada semente. De fato, em E. stipitata ssp. sororia McVaugh, espécie considerada monoembriônica, Anjos \& Ferraz (1999), realizando cortes nas sementes, observaram que, de 100 sementes seccionadas ao meio, nas metades que permaneceram com a zona meristemática obteve-se $89 \%$ de germinação e, ainda, $20 \%$ nas metades opostas à zona meristemática, o que totalizaria $109 \%$ de germinação.

Diante dos problemas citados e da possibilidade de se ampliar o número de plântulas obtidas de cada semente, este trabalho teve o objetivo de analisar os efeitos de diferentes formas de fracionamento das sementes de Eugenia pyriformis, relacionando sua eficiência com a procedência dos lotes, com a forma de coleta e com o tamanho das sementes, visando avaliar a capacidade regenerativa dessas sementes.

\section{Material e métodos}

Frutos maduros de uvaia (Eugenia pyriformis Cambess.) foram obtidos de quatro árvores de pomar instalado na Reserva Ecológica e Estação Experimental de Mogi-Guaçu, SP $\left(22^{\circ} 15-16^{\prime} \mathrm{S}\right.$ e $\left.47^{\circ} 8-12^{\prime} \mathrm{W}\right)$ e de duas árvores isoladas do Jardim Botânico de São Paulo, SP (233'' S e 46 37' W), em outubro de 1999. Em Mogi-Guaçu, foram coletados frutos ainda nas árvores e frutos já caídos ao solo, estes com sinais de queda recente. As sementes dos frutos de cada tipo de coleta foram mantidas separadas. Em São Paulo foram colhidos apenas frutos diretamente das árvores. A extração das sementes foi feita manualmente, com auxílio de peneira e água corrente. Após extração e lavagem, removeu-se o excesso de água da lavagem e as sementes foram separadas, através de peneira de crivo circular de 9,85 $\mathrm{mm}$ de diâmetro, em sementes graúdas e miúdas.

Tipo de coleta, tamanho e fracionamento das sementes Neste experimento foram utilizadas as sementes de Mogi-Guaçu, das árvores e do solo, graúdas e miúdas, que foram submetidas a quatro tipos de fracionamento: a) sementes intactas, ou seja, sem fracionamentos (figura 1), constituindo o controle; b) sementes fracionadas longitudinalmente ao meio, no sentido do maior diâmetro (figura 2), procurando-se passar o corte pelo hilo; c) sementes fracionadas transversalmente ao meio, ou seja, no sentido do menor diâmetro (figura 3), com o hilo permanecendo inteiro em uma das metades e d) fracionamento transversal, porém separando-se a semente em uma parte contendo aproximadamente $1 / 4$ da semente e a outra, que continha o hilo, ficando com $3 / 4$ do tamanho total da semente (figura 4). Todas as frações de sementes foram colocadas para germinar. Contudo, para cada repetição foram semeadas duas caixas (gerbox), uma para as frações que permaneceram com o hilo e outra para as demais.

Assim, foi constituído um experimento fatorial $2 \times 2 \times 4$ (tipo de coleta $\times$ tamanho das sementes $\times$ tipo de fracionamento nas sementes), em delineamento inteiramente casualizado com quatro repetições.

Tipos e número de fracionamentos e origem das sementes Neste experimento utilizaram-se as sementes de São Paulo, graúdas e miúdas, e as de Mogi-Guaçu, sem separação por tamanho. Nestas últimas, foram eliminadas as mais graúdas 
e as mais miúdas, formando-se um lote mais homogêneo de sementes de tamanho médio. As sementes desse segundo experimento foram colhidas diretamente nas árvores. Assim, foram formados três lotes de sementes, ou seja, graúdas de São Paulo, miúdas de São Paulo e medianas de Mogi-Guaçu.

Em cada um dos grupos, além das sementes que permaneceram intactas (controle), as sementes foram submetidas a fracionamentos, da seguinte forma: a) sementes fracionadas longitudinalmente em quatro partes (figura 5); b) sementes fracionadas transversalmente em quatro partes (figura 6); c) sementes fracionadas radialmente em quatro partes (figura 7) e d) sementes fracionadas radialmente em oito partes (figura 8). Assim como no experimento anterior, todas as frações de sementes foram colocadas para germinar. Neste experimento, porém, após os fracionamentos as frações de cada semente permaneceram agrupadas, no mesmo gerbox. Constituiu-se, dessa forma, um experimento fatorial $3 \times 4$ (lote $\times$ tipo de fracionamento realizado nas sementes).

As sementes, intactas ou fracionadas, foram colocadas para germinar em gerbox preenchido com vermiculita, com nove sementes por repetição, em germinadores com luz natural e temperatura regulada para $30^{\circ} \mathrm{C}$. Foram registrados, neste teste, o número de sementes (ou frações de sementes) que apresentaram protrusão de raiz primária, considerado para o cálculo da porcentagem de germinação, e o número de sementes (ou frações de sementes) que originaram plântulas normais (plântulas com sistema radicular presente e sem defeitos e epicótilo com folhas visíveis), considerado para o cálculo da porcentagem de sementes com capacidade de produzir plântulas normais. Foi calculado, também, o índice de velocidade de germinação (IVG), baseando-se na fórmula de Maguire (1962) e considerando-se a protrusão da raiz primária.

Em ambos os experimentos, a soma dos valores de germinação de todas as frações de cada semente foi utilizada para o cálculo do fator de incremento na germinação (FIG), ou seja, o número médio de frações que germinaram a partir do fracionamento de uma semente $\left(\mathrm{FIG}=\mathrm{nfg} \cdot \mathrm{ns}^{-1}\right.$, onde nfg é o número total de frações germinadas por repetição e ns é o número de sementes colocadas para germinar em cada repetição). Da mesma forma, a soma dos valores de plântulas normais obtidas de todas as frações de cada semente foi utilizada para o cálculo do fator de incremento na produção de plântulas normais (FIP), ou seja, o número médio de plântulas normais obtidas a partir do fracionamento de uma semente $\left(F I P=n p n . n s^{-1}\right.$, onde npn é o número total de plântulas normais por repetição e ns é o número de sementes colocadas para germinar em cada repetição).

Os resultados foram analisados estatisticamente pelo teste $\mathrm{F}, 5 \%$ de probabilidade, e as médias foram comparadas entre si pelo teste de Tukey (5\%). Os valores em porcentagem, para efeito de análise estatística, foram transformados para $\operatorname{arcsen}(\%)^{0,5}$.
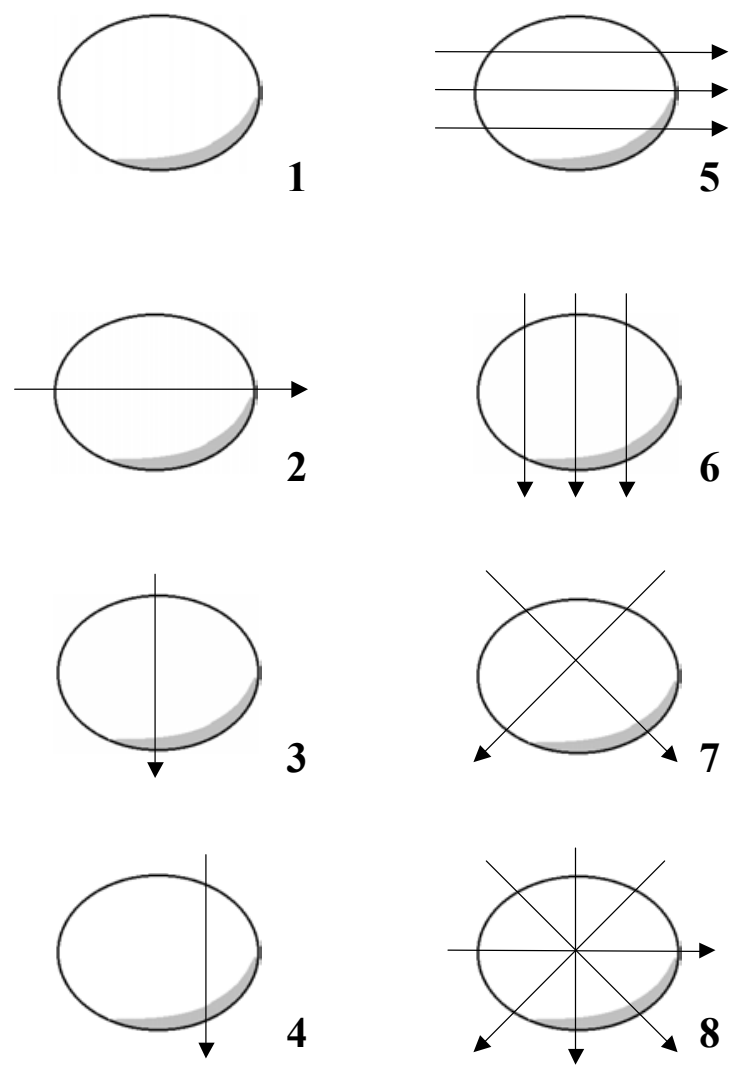

Figuras 1-8. Representação esquemática dos cortes efetuados nas sementes de Eugenia pyriformis Cambess. 1. Semente intacta. 2. Corte longitudinal ao meio. 3. Corte transversal ao meio. 4. Corte transversal separando uma parte contendo $3 / 4$ da semente e outra contendo $1 / 4$, com o hilo sempre nos $3 / 4$ de semente. 5. Cortes longitudinais separando a semente em quatro partes. 6. Corte transversal separando a semente em quatro partes. 7. Corte radial, separando a semente em quatro partes. 8. Corte radial, separando a semente em oito partes.

Figures 1-8. Outline of sections of Eugenia pyriformis Cambess. seeds. 1. Intact seed. 2. Seed sectioned longitudinally in the middle. 3 . Seed sectioned transversally in the middle. 4. Seed sectioned transversally resulting in one part (with the hilum) with $3 / 4^{\text {th }}$ of the seed and another part with $1 / 4^{\text {th }}$ of the seed. 5 . Seed sectioned longitudinally in 4 parts. 6. Seed sectioned transversally in 4 parts. 7. Seed sectioned radially in 4 parts. 8 . Seed sectioned radially in eight parts.

\section{Resultados e Discussão}

Tipo de coleta, tamanho e fracionamento das sementes - Considerando-se a germinação das sementes do controle (intactas), verificou-se que o tamanho e o tipo de coleta pouco afetaram o processo germinativo (tabela 1). A velocidade de germinação, medida pelo IVG, foi ligeiramente maior nas sementes obtidas de frutos coletados no solo, sugerindo que estas sementes 
Tabela 1. Germinação (\%) de sementes, desenvolvimento de plântulas normais (\%) e índice de velocidade de germinação (IVG) de sementes graúdas e miúdas de uvaia (Eugenia pyriformis Cambess.), obtidas de frutos coletados diretamente nas árvores ou no solo, em função de cortes transversais ou longitudinais. Germinação considerada apenas na fração da semente que permaneceu com o hilo.

Table 1. Seed germination (\%), development of normal seedlings (\%) and germination rate (IVG) of big and small seeds of "uvaia" (Eugenia pyriformis Cambess.), obtained from fruits collected directly from the trees or from the ground, longitudinally or transversally sectioned. Germination of the part that had the hilum.

\begin{tabular}{|c|c|c|c|c|c|}
\hline \multirow[t]{2}{*}{ Tipos de cortes } & \multicolumn{2}{|c|}{ Coleta na árvore } & \multicolumn{2}{|c|}{ Coleta no solo } & \multirow{2}{*}{ Médias } \\
\hline & Graúdas & Míúdas & Graúdas & Míúdas & \\
\hline & \multicolumn{5}{|c|}{ Germinação } \\
\hline Intactas & 98 a A $a^{1}$ & 100 a A $a$ & 100 a A $a$ & 98 a A $a$ & 99,0 \\
\hline Meio, longitudinal & 98 a A $a$ & 89 ab A $a$ & 98 a A $a$ & 93 a A $a$ & 94,5 \\
\hline Meio, transversal & 94 a A $a$ & $82 \mathrm{~b} \mathrm{~B} b$ & $77 \mathrm{~b} \mathrm{~B} b$ & 93 a A $a$ & 86,5 \\
\hline $3 / 4$, transversal & 92 a A $a$ & 98 a A $a$ & 95 a A $a$ & 92 a A $a$ & 94,2 \\
\hline Médias & 95,5 & 92,2 & 92,5 & 94,0 & \\
\hline \multirow[t]{2}{*}{ C.V. $(\%)$} & 7,75 & & & & \\
\hline & \multicolumn{5}{|c|}{ Plântulas normais } \\
\hline Intactas & 96 & 97 & 100 & 97 & $97,4 \mathrm{a}$ \\
\hline Meio, longitudinal & 69 & 68 & 78 & 78 & $73,3 \mathrm{bc}$ \\
\hline Meio, transversal & 73 & 66 & 62 & 67 & $66,8 \mathrm{c}$ \\
\hline $3 / 4$, transversal & 78 & 80 & 81 & 76 & $78,9 \mathrm{~b}$ \\
\hline Médias & $78,8 \mathrm{~A}$ & $76,9 \mathrm{~A}$ & $80,1 \mathrm{~A}$ & $80,6 \mathrm{~A}$ & \\
\hline \multirow[t]{2}{*}{ C.V. $(\%)$} & 13,05 & & & & \\
\hline & \multicolumn{5}{|c|}{ IVG } \\
\hline Intactas & 0,609 & 0,539 & 0,699 & 0,647 & $0,623 \mathrm{a}$ \\
\hline Meio, longitudinal & 0,430 & 0,353 & 0,459 & 0,445 & $0,422 b$ \\
\hline Meio, transversal & 0,412 & 0,282 & 0,395 & 0,429 & $0,380 \mathrm{~b}$ \\
\hline 3/4, transversal & 0,454 & 0,415 & 0,511 & 0,502 & $0,470 \mathrm{~b}$ \\
\hline Médias & \multicolumn{2}{|c|}{$0,437 b$} & \multicolumn{2}{|c|}{$0,511 a$} & \\
\hline C.V. $(\%)$ & 21,88 & & & & \\
\hline
\end{tabular}

${ }^{1}$ Médias seguidas pela mesma letra não diferem entre si pelo teste de Tukey ao nível de 5\% de probabilidade. Minúsculas para comparações nas colunas; maiúsculas nas linhas, dentro de coletas; itálicas nas linhas, entre coletas.

poderiam estar em estádio mais avançado no processo de maturação.

Os resultados obtidos demonstraram a elevada capacidade germinativa das sementes de Eugenia pyriformis, mesmo quando danificadas. Durante a seleção de sementes danificadas do lote, observaram-se várias com lesões causadas por insetos ou pássaros, algumas vezes tomando cerca de metade das sementes, que foram eliminadas. Contudo, o efeito de danos físicos à semente poderia ser comparado ao fracionamento realizado neste experimento (figura 9), que resultou em germinação sempre superior a $77 \%$ (tabela 1), com desenvolvimento de plântulas normais até nas frações que permaneceram com apenas $1 / 4$ da semente (figura 10).

A eliminação de metade da semente graúda por meio de fracionamento longitudinal não afetou seu potencial germinativo ( $98 \%$, tabela 1), mas resultou, como era esperado, em pequena redução na capacidade de produzir plântulas normais (69-78\%, tabela 1). Por outro lado, para as sementes miúdas verificou-se redução tanto na germinação quanto na capacidade de produzir plântulas normais. Contudo, nesse tipo de fracionamento a capacidade de produção de plântulas normais manteve-se sempre acima de $68 \%$ (tabela 1), inclusive para as sementes miúdas.

Quando o fracionamento das sementes foi realizado no sentido do menor diâmetro (transversal), a redução no potencial germinativo e na capacidade de produção de plântulas normais variou em função do tamanho da fração removida. Quando foi removido apenas $1 / 4$ da semente, os valores de germinação e de produção de plântulas normais foram semelhantes aos das seccionadas longitudinalmente (tabela 1). Por outro 
lado, quando se removeu metade da semente houve maior prejuízo, principalmente na produção de plântulas normais, chegando-se a valores de $77 \%$ de germinação e $62 \%$ de sementes produzindo plântulas normais. Estes resultados evidenciam que as sementes de Eugenia pyriformis são mais prejudicadas quando sofrem lesões perpendiculares ao seu maior eixo.

Em algumas espécies de Eugenia, o tegumento exerce influência sobre a germinação da semente, freqüentemente ocasionando atraso (Rizzini 1970, Gentil \& Ferreira 1999). Em E. pyriformis, porém, sementes intactas apresentaram germinação rápida, com valores de IVG superiores a 0,5 (tabela 1 ), praticamente duas vezes maiores que o observado para E. stipitata ssp. sororia por Gentil \& Ferreira (1999). Os resultados do IVG demonstraram que houve redução na velocidade de germinação das sementes submetidas a qualquer tipo de fracionamento (tabela 1).

Conforme descrito anteriormente, as frações das sementes que não permaneceram com o hilo também foram colocadas para germinar, inclusive as frações que permaneceram com apenas $1 / 4$ da semente. Os valores de germinação e de desenvolvimento de plântulas normais destas frações foram somados aos da tabela 1 para a obtenção do fator de incremento no número médio de frações germinadas por semente (FIG) e do fator de incremento no número de plântulas normais obtidas de cada semente (FIP), que estão apresentados na tabela 2. O FIG e o FIP das sementes intactas não superou 1,00 (tabela 2), ou seja, não houve mais do que um embrião germinando por semente, o que era esperado pois, segundo Gurgel \& Soubihe Sobrinho (1951), não possuem poliembrionia. Por outro lado, o FIG e o FIP das sementes fracionadas foram quase sempre superiores a 1,00 (tabela 2), indicando elevada capacidade regenerativa.

O surgimento de mais de uma plântula normal a partir de uma única semente poderia sugerir algum grau de poliembrionia em Euglena pyriformis. Rizzini (1970) verificou que sementes de E. dysenterica têm substâncias inibidoras da germinação e que o potencial inibidor dessas substâncias aumenta quando o embrião começa a germinar e diminui quando há contato do inibidor com o ar. Se esse efeito ocorresse em Euglena pyriformis, seria difícil a identificação de poliembrionia, pois um embrião poderia exercer inibição sobre outros. Os fracionamentos, nesse caso, poderiam estar anulando o efeito dos inibidores. A identificação de poliembrionia em E. pyriformis poderia ser prejudicada, também, caso as sementes contenham embriões coalescidos, como observado em
E. hookeri Steud. (Johnson 1936), dificultando a visualização de todos os embriões existentes na semente. O estudo de substâncias inibidoras da germinação em sementes de E. pyriformis poderia contribuir para elucidar essa hipótese, assim como o fracionamento de sementes de E. dysenterica, monoembriônicas segundo Salomão \& Allem (2001).

Outra hipótese para a existência de mais de uma plântula a partir da mesma semente seria que, na verdade, um único eixo embrionário estaria sendo fracionado e cada uma de suas frações resultantes teria a capacidade de regenerar uma nova plântula. Conforme descrito por Lughadha \& Proença (1996), Tiwary, em 1926, considerou poliembriônicas as sementes de espécies de Eugenia que, na verdade, apresentaram bifurcação da plúmula. De fato, em algumas sementes fracionadas de E. pyriformis notou-se a formação de dois epicótilos e dois sistemas radiculares, unidos pelo colo, a partir de uma mesma fração de semente (figura 11). Tal fenômeno não foi observado em nenhuma das sementes intactas.

Uma terceira hipótese, ainda, poderia ser que uma das frações da semente fracionada passaria a ter comportamento semelhante a um caule, com migração de hormônios e formação de calos meristemáticos regenerativos com capacidade de produzir novas plântulas, conforme observado por Anjos \& Ferraz (1999) em sementes de Eugenia stipitata ssp. sororia. Nesse caso, frações menores, como a que permaneceu com apenas $1 / 4$ da semente, poderiam ter maior dificuldade em produzir plântulas normais pela insuficiência de reserva nutritiva.

Seja qual for a razão da formação dessas plântulas, o fracionamento das sementes de E. pyriformis pode quase duplicar a porcentagem de germinação e se obter $50 \%$ a mais de plântulas normais (tabela 2 ).

Os valores de FIG próximos a 1,5 das sementes fracionadas transversalmente a $3 / 4$ (tabela 2) indicam que cerca de $50 \%$ das frações com apenas $1 / 4$ da semente ainda apresentavam capacidade de germinar, algumas produzindo plântulas com todas as estruturas essenciais (figuras 9 e 10). Contudo, verifica-se pelos valores de FIP que a redução no tecido de reserva dificultou o desenvolvimento de plântulas normais. É importante salientar que, nas frações de sementes com produção de duas plântulas (figura 11), que ocorreu apenas em sementes fracionadas, apenas uma das plântulas foi registrada para o cálculo da porcentagem de germinação, de desenvolvimento de plântulas normais, do FIG e do FIP.

Tipos e número de fracionamentos e origem das 

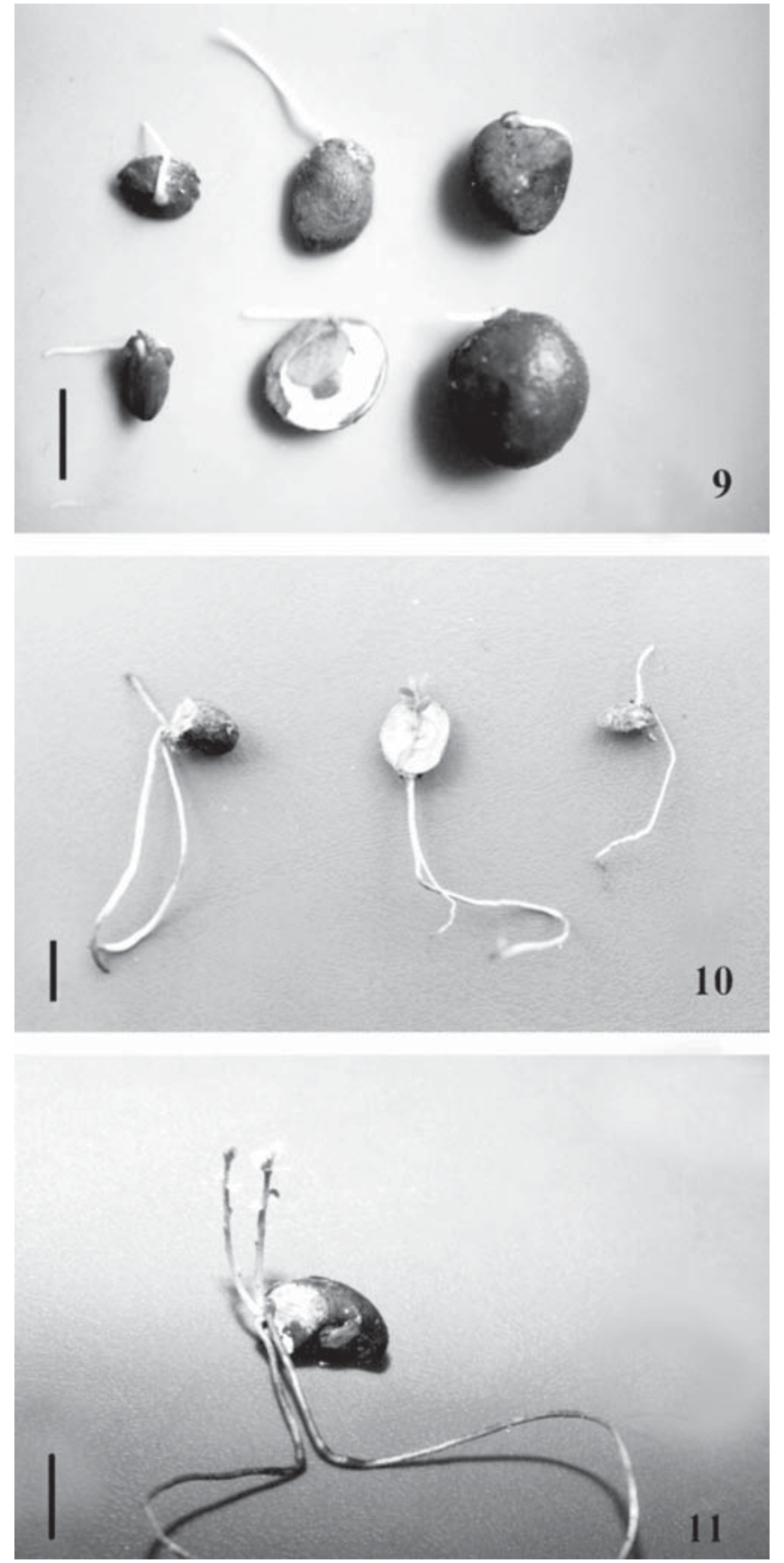

Figuras 9-11. Germinação de sementes e frações de sementes de Eugenia pyriformis Cambess. 9. Protrusão da raiz primária e desenvolvimento inicial da raiz primária. À direita, semente intacta; ao centro, semente fracionada ao meio longitudinalmente; à esquerda, 1/4 de semente fracionada transversalmente. 10. Plântulas normais. À esquerda, plântula obtida de $3 / 4$ de semente fracionada transversalmente; ao centro, plântula normal obtida de metade de semente fracionada longitudinalmente; à direita, plântula normal obtida de 1/4 de semente fracionada transversalmente. 11 . Desenvolvimento de duas plântulas normais a partir da mesma fração de semente fracionada transversalmente a $3 / 4$. As duas plântulas permaneceram fundidas pelo colo. Escalas $=1 \mathrm{~cm}$. sementes - Os resultados obtidos no experimento anterior, indicando a capacidade regenerativa das sementes de Eugenia pyriformis, suscitaram a dúvida quanto ao limite de fracionamento que estas sementes suportariam e, ainda, se tal efeito também ocorreria com sementes de outra região. Assim, elevou-se o limite de fracionamentos até a produção de oito frações a partir de uma única semente, empregando-se sementes de Mogi-Guaçu e de São Paulo.

As sementes intactas (controle) de ambos os lotes (São Paulo e Mogi-Guaçu) apresentaram elevados valores de germinação e de desenvolvimento de plântulas normais (FIG e FIP superiores a 0,90 e 0,85, respectivamente) e não apresentaram germinação superior a $100 \%$ (figura 12 ).

As frações obtidas do fracionamento transversal em quatro partes (figura 6) poderiam ser comparadas à fração de $1 / 4$ de semente obtida no experimento anterior (figura 4). FIG e FIP, no primeiro experimento, demonstrando que aproximadamente $50 \%$ das frações com $1 / 4$ da semente ainda apresentavam capacidade germinativa (tabela 2), foram muito próximos aos obtidos com o fracionamento em quatro partes, transversalmente, que originou FIG e FIP de, respectivamente, 1,60 e 0,95 . Contudo, neste último caso, não havia frações com $3 / 4$ da semente e todas tinham apenas $1 / 4$.

A influência do tamanho da fração resultante ficou evidente nas sementes miúdas de São Paulo, que apresentaram desempenho germinativo inferior ao das graúdas (figura 12). A importância da posição do fracionamento ficou mais evidente comparando-se os resultados do fracionamento transversal com os do longitudinal. Neste último, para sementes de Mogi-Guaçu, obteve-se FIG de 2,20 contra 1,60 daquele, embora os FIPs tenham sido semelhantes $(1,00$ contra 0,95 , figura 12 ).

Comparando-se os valores de sementes graúdas e

Figures 9-11. Germination of seeds and parts of seeds of Eugenia pyriformis Cambess. 9. Protrusion of radicle and initial development of radicle. On the right, intact seed; in the middle, seed sectioned longitudinally in the middle; on the left, $1 / 4^{\text {th }}$ of the seed sectioned transversally. 10 . Normal seedlings. On the left, seedling from $3 / 4^{\text {th }}$ of the seed sectioned transversally; in the middle, normal seedling from half part of a seed sectioned longitudinally; on the right, normal seedling from $1 / 4^{\text {th }}$ of the seed sectioned transversally. 11. Development of two normal seedlings from $3 / 4^{\text {th }}$ of the seed sectioned transversally. The two seedlings remained fused by the base of the stem. Scale $=1 \mathrm{~cm}$. 
Tabela 2. Fator de incremento na germinação (FIG) e na produção de plântulas normais (FIP) por semente, de sementes graúdas e miúdas de uvaia (Eugenia pyriformis Cambess.), coletadas diretamente nas árvores ou recolhidas do solo imediatamente após a queda e colocadas para germinar íntegras ou cortadas ao meio em diferentes seções.

Table 2. Germination increase factor (FIG) and in normal seedlings production (FIP) from each big or small seed of "uvaia" (Eugenia pyriformis Cambess.), obtained from fruits collected directly from the trees or from the ground, sectioned or not.

\begin{tabular}{|c|c|c|c|c|c|}
\hline \multirow[t]{2}{*}{ Tipos de cortes } & \multicolumn{2}{|c|}{ Coleta na árvore } & \multicolumn{2}{|c|}{ Coleta no solo } & \multirow[t]{2}{*}{ Médias } \\
\hline & Graúdas & Míúdas & Graúdas & Miúdas & \\
\hline & \multicolumn{5}{|c|}{$\mathrm{FIG}$} \\
\hline Intactas & 1,00 & 1,00 & 0,98 & 0,98 & $0,99 \mathrm{~d}^{1}$ \\
\hline Meio, longitudinal & 1,90 & 1,74 & 1,94 & 1,78 & $1,84 \mathrm{a}$ \\
\hline Meio, transversal & 1,48 & 1,51 & 1,64 & 1,83 & $1,61 \mathrm{~b}$ \\
\hline $3 / 4$, transversal & 1,50 & 1,47 & 1,45 & 1,45 & $1,47 \mathrm{c}$ \\
\hline Médias & \multicolumn{2}{|c|}{$1,48 \mathrm{~A}$} & \multirow{2}{*}{\multicolumn{2}{|c|}{$1,47 \mathrm{~A}$}} & \\
\hline \multirow[t]{2}{*}{ C.V. $(\%)$} & 9,77 & & & & \\
\hline & \multicolumn{5}{|c|}{ FIP } \\
\hline Intactas & 0,96 & 0,97 & 1,00 & 0,97 & $0,97 \mathrm{c}$ \\
\hline Meio, longitudinal & 1,32 & 1,22 & 1,46 & 1,41 & $1,35 \mathrm{a}$ \\
\hline Meio, transversal & 1,26 & 1,15 & 1,12 & 1,25 & $1,19 \mathrm{~b}$ \\
\hline $3 / 4$, transversal & 1,18 & 1,06 & 1,09 & 1,00 & $1,08 \mathrm{bc}$ \\
\hline Médias & \multicolumn{2}{|c|}{$1,14 \mathrm{~A}$} & \multicolumn{2}{|c|}{$1,16 \mathrm{~A}$} & \\
\hline C.V. $(\%)$ & 12,72 & & & & \\
\hline
\end{tabular}

${ }^{1}$ Médias seguidas pela mesma letra não diferem entre si pelo teste de Tukey ao nível de 5\% de probabilidade. Minúsculas para comparações nas colunas; maiúsculas nas linhas.

miúdas de São Paulo verifica-se a importância tanto do tamanho quanto da posição do fracionamento. As sementes graúdas que, no fracionamento transversal, tiveram FIG e FIP de 1,80 e 1,00, respectivamente, apresentaram 3,50 e 1,70 no fracionamento longitudinal (o que corresponderia a 350\% de germinação e $170 \%$ de produção de plântulas normais). Contudo, considerando-se as sementes miúdas verifica-se que, embora o FIG tenha sido 2,50 (figura 12-A), o FIP foi 1,00 (figura 12-B), ou seja, não se obteve mais do que uma plântula normal por semente, portanto não apresentando vantagens em fracioná-las.

A elevada capacidade regenerativa das sementes de Eugenia pyriformis ficou evidente nos fracionamentos radiais, em quatro ou em oito partes. Em ambos os casos, para sementes de Mogi-Guaçu, obtiveram-se FIG e FIP nunca inferiores a, respectivamente, 1,50 e 0,90 (figura 12). Assim, fracionando-se a semente em até oito frações, ao menos uma das frações obtidas ainda pode produzir uma plântula normal. Nas sementes graúdas de São Paulo, os valores de FIG e FIP para sementes fracionadas em oito partes foram de 2,40 e 0,90 , respectivamente (figura 12), ou seja, $30 \%$ das frações com $1 / 8$ da semente apresentaram capacidade de germinar e aproximadamente $11 \%$ das frações obtidas puderam produzir plântula normal.

Os valores de FIG e FIP foram sempre superiores no fracionamento longitudinal, seguido pelo radial e, por último, o transversal (figura 12). Assim, fica evidente que o fracionamento transversal é o que mais prejudica a capacidade germinativa das sementes de Eugenia pyriformis.

Os resultados obtidos nos dois experimentos permitem inferir que, embora possível, é pouco provável que as sementes de Eugenia pyriformis sejam poliembriônicas como outras do gênero Eugenia (Johnson 1936, Gurgel \& Soubihe Sobrinho 1951, Lughadha \& Proença 1996) ou, ainda, que a obtenção de mais de uma plântula a partir dos fracionamentos seja oriunda de bifurcações da plúmula (Lughadha \& Proença 1996). A probabilidade de que os fracionamentos tivessem sido realizados, todos ou na sua grande maioria, na região de separação dos embriões ou na metade dos eixos embrionários é muito baixa. Reforça-se, portanto, a possibilidade de frações de sementes assumirem comportamento semelhante ao de estacas, com possibilidade de enraizamento e desenvolvimento de nova parte aérea, a partir da migração de hormônios vegetais (Anjos \& Ferraz 1999). 

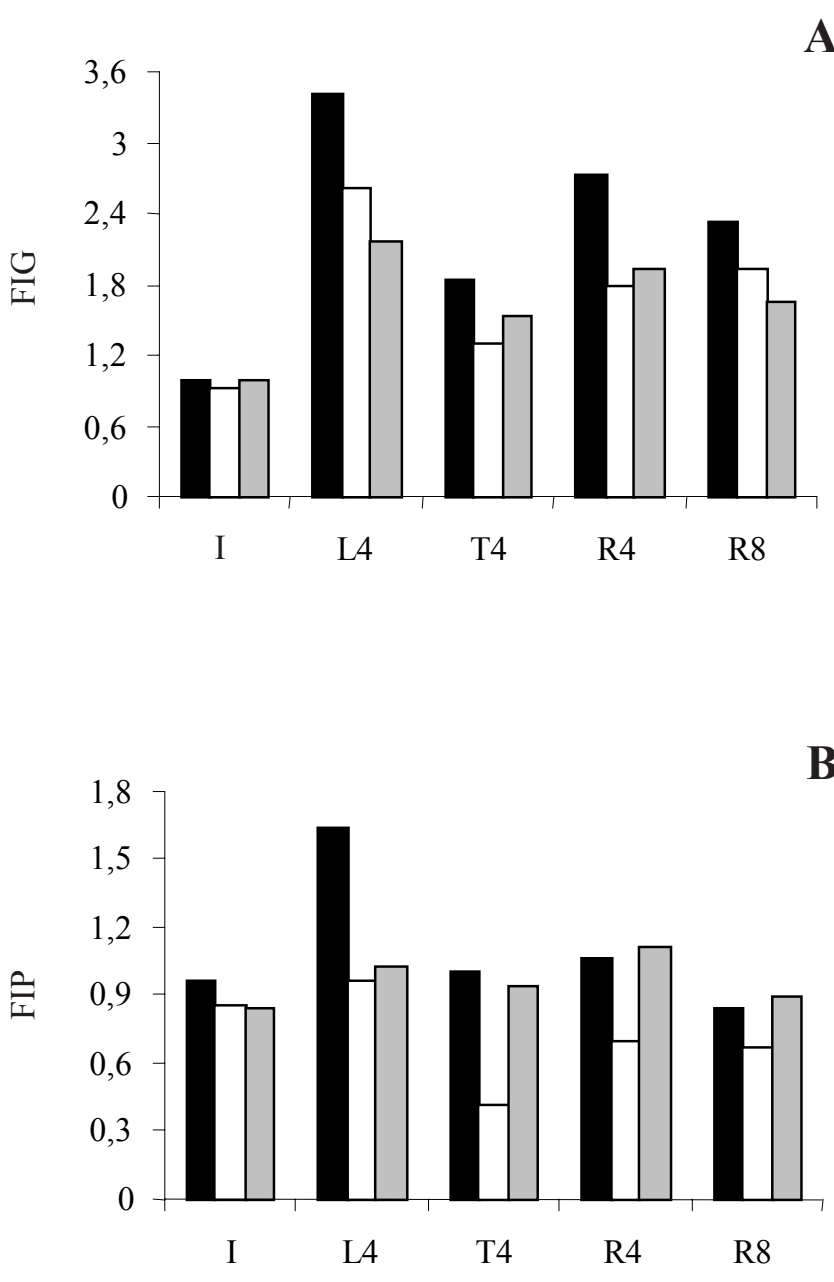

Figura 12. Fator de incremento na germinação (FIG) e na produção de plântulas normais (FIP) por semente de Eugenia pyriformis Cambess. submetidas a diferentes formas de corte. A) FIG: Diferença mínima significativa (DMS) $)_{5 \%}$ cortes $=$ 0,6554; $\mathrm{DMS}_{5 \%}$ lotes =0,5585; B) FIP: $\mathrm{DMS}_{5 \%}$ cortes =0,3978; $\mathrm{DMS}_{50}$ lotes $=0,3390$. Coluna preta: sementes graúdas do lote São Paulo; coluna branca: sementes miúdas do lote São Paulo; coluna cinza: lote Mogi-Guaçu. I = sementes intactas; L4 = sementes fracionadas longitudinalmente em quatro partes; $\mathrm{T} 4=$ sementes fracionadas transversalmente em quatro partes; R4 = sementes fracionadas radialmente em quatro partes; $\mathrm{R} 8=$ sementes fracionadas radialmente em oito partes.

Figure 12. Germination increase factor (FIG) and normal seedlings production factor (FIP) from seed of Eugenia pyriformis Cambess. sectioned by different methods. A) FIG: Minimum significant difference $(\mathrm{MSD})_{5 \%}$ section $=0.6554$; $\mathrm{MSD}_{5 \%}$ lots $=0.5585$; B) FIP: $\mathrm{MSD}_{5 \%}$ section $=0.3978 ; \mathrm{MSD}_{5 \%}$ lots $=0.3390$. Black columns: large seeds from São Paulo; white columns: small seeds from São Paulo; gray columns: seeds from Mogi-Guaçu. I = intact seeds; L4 = seeds sectioned longitudinally in four parts; T4 = seeds sectioned transversally in four parts; R4 = seeds sectioned radially in four parts; R8 $=$ seeds sectioned radially in eight parts.
Estudos de ontogenia e de desenvolvimento de frutos e sementes, como os realizados para Campomanesia (Landrum 1982) e para espécies de Eugenia da África (Van Wyk \& Botha 1984), bem como a análise da anatomia das sementes maduras, serão fundamentais para a melhor compreensão do fenômeno.

De qualquer forma, é interessante registrar as implicações ecológicas e práticas do potencial regenerativo das sementes de uvaia. Por um lado, uma vez que insetos, aves e mamíferos são atraídos pelos frutos dessa espécie, muitas vezes causando injúrias às sementes, a capacidade regenerativa demonstrada pelos resultados do presente trabalho conferem alguma segurança para a reprodução, dispersão e propagação da espécie. Por outro lado, levando-se em conta a possibilidade de ocorrência de condições ambientais adversas imediatamente após a germinação das sementes, haveria reserva nutritiva e capacidade regenerativa suficientes para germinação e desenvolvimento de algumas novas plântulas a partir da mesma semente. Finalmente, considerando-se o interesse agronômico e a dificuldade de obtenção de mudas de Eugenia pyriformis, fica evidenciada a possibilidade de, com fracionamentos longitudinais ao seu maior eixo, ampliar a produção de mudas a partir de um mesmo lote de sementes.

Agradecimentos - aos funcionários da Fazenda Campininha, em Mogi-Guaçu, pela coleta de frutos; ao CNPq, pela bolsa de produtividade concedida a C.J. Barbedo; a José Aparecido da Silva, pelo tratamento eletrônico das imagens fotográficas; a Adeliana S.C. Barbedo, pelas valiosas sugestões na revisão do manuscrito.

\section{Referências bibliográficas}

ANJOS, A.M.G. \& FERRAZ, I.D.K. 1999. Morfologia, germinação e teor de água das sementes de araçá-boi (Eugenia stipitata ssp. sororia). Acta Amazonica 29:337-348.

BARBEDO, C.J., KOHAMA, S., MALUF, A.M. \& BILIA, D.A.C. 1998. Germinação e armazenamento de diásporos de cerejeira (Eugenia involucrata DC. Myrtaceae) em função do teor de água. Revista Brasileira de Sementes 20:184-188.

CONSOLINI, A.E., BALDINI, O.A.N. \& AMAT, A.G. 1999. Pharmacological basis for the empirical use of Eugenia uniflora L. (Myrtaceae) as anthypertensive. Journal of Ethnopharmacology 66:33-39.

CORRÊA, M.P. 1975. Dicionário da plantas úteis do Brasil e das exóticas cultivadas. Ministério da Agricultura, Rio de Janeiro. 
FLORES, E.M. \& RIVERA, D.I. 1989. Criptocotilia em algumas dicotiledoneas tropicales. Brenesia 32:19-26.

GENTIL, D.F.O. \& FERREIRA, S.A.N. 1999. Viabilidade e superação da dormência em sementes de araçá-boi (Eugenia stipitata ssp. sororia). Acta Amazonica 29:21-31.

GURGEL, J.T.A. \& SOUBIHE SOBRINHO, J. 1951. Poliembrionia em mirtáceas frutíferas. Bragantia 11:141-163.

JOHNSON, A.M. 1936. Polyembryony in Eugenia hookeri. American Journal of Botany 23:83-88.

KAWASAKI, M.L. 2000. Flora fanerogâmica do Parque Estadual das Fontes do Ipiranga (São Paulo, Brasil). 88 - Myrtaceae. Hoehnea 27:165-186.

LANDRUM, L.R. 1982. The development of the fruits and seeds of Campomanesia (Myrtaceae). Brittonia 34:220-224.

LANDRUM, L.R. \& KAWASAKI, M.L. 1997. The genera of Myrtaceae in Brazil: an illustrated synoptic treatment and identification keys. Brittonia 49:508-536.

LUGHADHA, E.N. \& PROENÇA, C. 1996. A survey of the reproductive biology of the Myrtoideae (Myrtaceae). Annals of the Missouri Botanical Garden 83:480-503.

LUNARDI, I., PEIXOTO, J.L.B., SILVA, C.C., SHUQUEL, I.T.A., BASSO, E.A. \& VIDOTTI, G.J. 2001. Triterpenic acids from Eugenia moraviana. Journal of Brazilian Chemical Society 12:180-183.

MAGUIRE, J.D. 1962. Speed of germination-aid in selection and evalution for seedling emergence vigor. Crop Science 2:176-177.

MATTOS, J.R. 1954. Estudo pomológico dos frutos indígenas do Rio Grande do Sul. Oficinas Gráficas da Imprensa Oficial, Porto Alegre.

MCVAUGH, R. 1956. Tropical American Myrtaceae. Fieldiana Botany 29:143-228.

NASCIMENTO, V.M., XAVIER, A.A., CORRÊA, L.S. \& VALÉRIO FILHO, W.V. 1995. Physical and chemical characteristics of the fruit of native's species on the Brazilian cerrado. Acta Horticulturae 370:113-116.
PEPATO, M.T., FOLGADO, V.B.B., KETTELHUT, I.C. \& BRUNETTI, I.L. 2001. Lack of antidiabetic effect of a Eugenia jambolana leaf decoction on rat streptozotocin diabetes. Brazilian Journal of Medical and Biological Research 34:389-395.

RIZZINI, C.T. 1970. Efeito tegumentar na germinação de Eugenia dysenterica DC. (Myrtaceae). Revista Brasileira de Biologia 30:381-402.

SALOMÃO, A.N. \& ALLEM, A.C. 2001. Polyembryony in Angiospermous trees of the Brazilian cerrado and caatinga vegetation. Acta Botanica Brasilica 15:369-378.

SCHMEDA-HIRSCHMANN, G., THEODULOZ, C., FRANCO, L., FERRO, E.B. \& ARIAS, A.R. 1987. Preliminary pharmacological studies on Eugenia uniflora leaves: xanthine oxidase inhibitory activity. Journal of Ethnopharmacology 21:183-186.

SCHMID, R. 1972. A resolution of the Eugenia-Syzygyum controversy (Myrtaceae). American Journal of Botany 59:423-436.

SILVA, R.S.M., CHAVES, L.J. \& NAVES, R.V. 2001. Caracterização de frutos e árvores de cagaita (Eugenia dysenterica DC.) no sudeste do Estado de Goiás, Brasil. Revista Brasileira de Fruticultura 23:330-334.

THEODUlOZ, C., FRANCO, L., FERRO, E.B. \& SCHMEDA-HIRSCHMANN, G. 1988. Xanthine oxidase inhibitory activity of Paraguayan Myrtaceae. Journal of Ethnopharmacology 24:179-183.

VAN WYK, A.E. \& BOTHA, R. 1984. The genus Eugenia (Myrtaceae) in southern Africa: ontogeny and taxonomic value of the seed. South African Journal of Botany 3:63-80.

VON BÜLOW, J.F.W., CARMONA, R. \& VAZ PARENTE, T. 1994. Armazenamento e tratamento de sementes de pitanga-vermelha-do-cerrado (Eugenia calycina). Pesquisa Agropecuária Brasileira 29:961-970. 